\title{
FEATURE ANALYSIS FOR MULTI-WINDOW MATCHING
}

\author{
W. C. Chang ${ }^{\mathrm{a}, *}$, L. C. Chen ${ }^{\mathrm{b}}$ \\ ${ }^{a}$ Dept. of Civil Engineering, National Central University, Taiwan-wenchi@ csrsr.ncu.edu.tw \\ ${ }^{\mathrm{b}}$ Center for Space and Remote Sensing Research, National Central University, Taiwan - lcchen@ csrsr.ncu.edu.tw
}

\section{Youth Forum}

KEY WORDS: Matching, Feature, Aerial, Image, Photogrammetry

\begin{abstract}
:
Image matching is a practical way to build up the relationship between point pairs. In remote sensing and photogrammetry applications, area-based matching algorithms are usually used under control environment, such as fixing exterior orientation parameters, to reach high matching precision. In order to improve the reliability of image matching, area-based matching algorithms are frequently performed with image pyramid and epipolar constraints. Thus, Central-Left-Right matching (CLR matching) is proposed to enhance the reliability. The method is designed to cope with unreliable matching when the objects are with surface discontinuity. The CLR matching puts a candidate point in the center, left and right windows. Thus, the elements of target window and search window may be corresponded to the same objects if the candidate point is located in a vertical edge. In addition, the directions of features in the image may be varied because of the object diversity. Thus, the CLR matching would be better to combine with multi-window operation in accordance with the feature direction. This paper analyzed the feature direction first followed by the multi-window matching. The major works in this investigation contain feature extraction, feature analysis, and image matching. The experiments compared the results of traditional image matching, CLR matching, and multi-window matching. The experimental results indicate that the proposed method improves the accuracy of image matching.
\end{abstract}

\section{INTRODUCTION}

The determination of conjugate point pairs is important in geometric correction, image mosaicking, and three dimensional object modeling, etc. Image matching is a practical way to build up the relationship between those point pairs. According to its strategy, image matching can be divided into three categories, namely feature-based matching, area-based matching, and relational matching (Rosenholm, 1987; Habib, 2003). Featurebased matching algorithms are widely used for computer vision, pattern recognition, and medical image registration. In remote sensing and photogrammetry applications, area-based matching algorithms are usually used under control environment (Xiong and Zhang, 2009), such as fixing exterior orientation parameters, to reach high matching precision.

In order to improve the reliability of image matching, areabased matching algorithms are frequently performed with image pyramid and epipolar constraints. Thus, Central-Left-Right matching (CLR matching) is proposed to enhance the reliability, especially for the area with elevation discontinuity (Hsu, 1990). For building boundaries, as a result of the elevation discontinuity between buildings and ground in aerial images, different view angles may lead to ambiguities in image matching because the elements around the feature point may cover different backgrounds. The CLR matching puts a candidate point in the center, left and right windows. Thus, the elements of target window and search window may be corresponded to the same objects if the candidate point is located in a vertical edge, such as Figure 1(A).

However, the directions of features in the image are varied because of the object diversity. The CLR matching should become multi-window operation in accordance with the feature direction. Figure 1(B), for example, the candidate point should be putting along the feature direction instead of center, left and right windows. Thus, this paper improves the CLR matching for aerial images. We analyze the feature direction first followed by the multi-window matching.

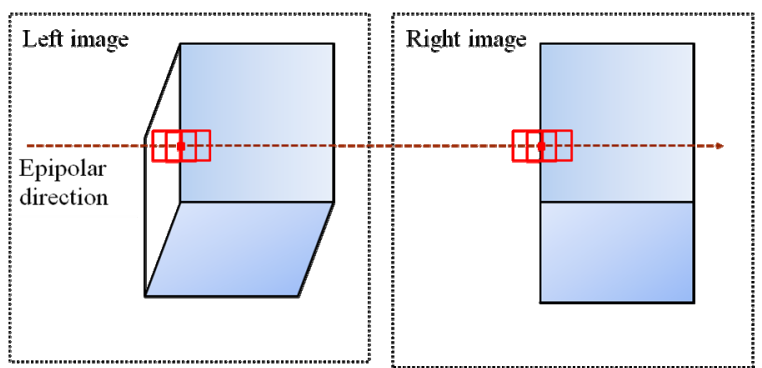

(A) CLR matching

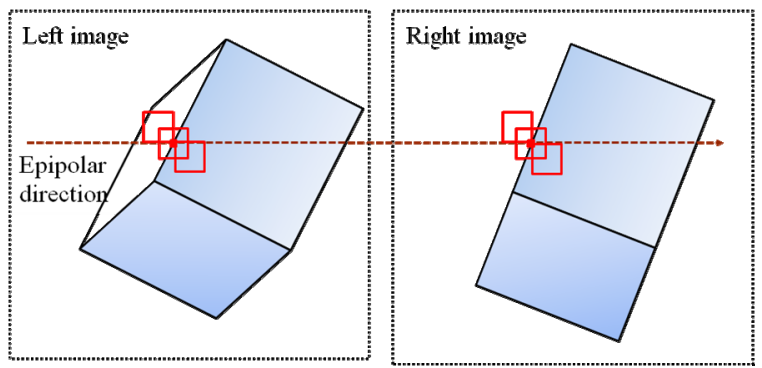

(B) Multi-window matching

Figure 1. Illumination of the difference between CLR matching and Multi-window matching

\footnotetext{
* Corresponding author. This is useful to know for communication with the appropriate person in cases with more than one author.
} 


\section{METEODOLOGY}

The major works in this investigation contain feature extraction, feature analysis, and image matching. The workflow is shown in Figure 2. We choose the image with the closest to nadir point as the master image, and others are slave images. The details of each part are given below.

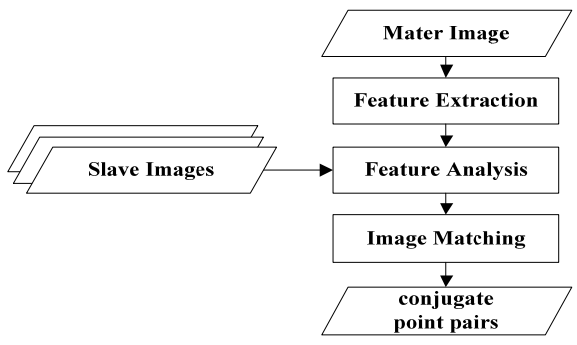

Figure 2. Workflow of the proposed scheme

\subsection{Feature Extraction}

Considering the feature direction, this paper employs the line features for image matching. Canny edge detection (Canny, 1986) is performed to extract line features in working area. Those edge pixels are considered as candidate point to do feature analysis and image matching.

\subsection{Feature Analysis}

In this step, we decide the multi-window directions for each candidate point. This paper sets four multi-window directions types, as shown in Figure 3, for image matching.

After feature extraction, the edge points are divided into two categories, namely point feature and straight line feature. Hough transform (Hough, 1959) is selected for determined the straight line features. And the remaining points are deemed as point features.

For point features, the corresponding feature direction is determined by the gradient of grey value. For each straight line feature, we calculate the corresponding feature direction in the Hough space. According to the feature direction, we assign the multi-window direction type with the closest values to feature direction.
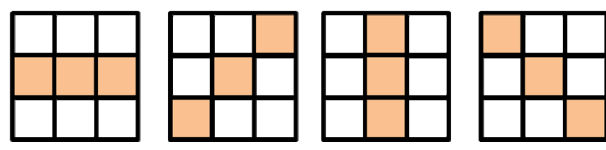

Figure 2. Multi-window Directions

\subsection{Image Matching}

This paper selects the object-based matching method (Zhang and Gruen, 2006) for image matching. Starting from a feature point in the master image, the method connects the correspondences in different images through a same object location. We generate the corresponding object coordinates by ray tracing with an initial elevation. Then the object coordinates are back projected to the other images to obtain the search windows. The weighted mean of correlation coefficients are calculated for a target window in the master image and the search windows.
The steps will be performed iteratively with different elevations in a setting height range until converged. The final correspondences for this feature point are determined by the maximum average correlation coefficient.

Besides, the multi-window strategy is included in the matching procedure. In the object-based image matching, each target window and search window will selected by the multiple windows that are determined by feature analysis.

\section{EXPERIMENTS}

The test data set includes five Digital Mapping Camera II (DMC II) images with about $10 \mathrm{~cm}$ spatial resolution. The other information about test images shows in Table 1. Figure 3 shows the aerial image of a test example.

\begin{tabular}{|c|c|}
\hline Camera & RMK DX \\
\hline Date & 10 Mar 2010 \\
\hline Focal length & $91.9817 \mathrm{~mm}$ \\
\hline Image format & $12096 \times 11200$ pixels \\
& $97.2 \mu \mathrm{m}$ \\
& $10 \mathrm{~cm}$ \\
\hline Spatial resolution & \\
\hline
\end{tabular}

Table 1. Information Related to Test Image

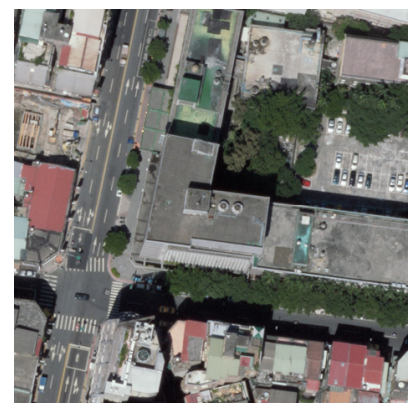

(A) Master Image

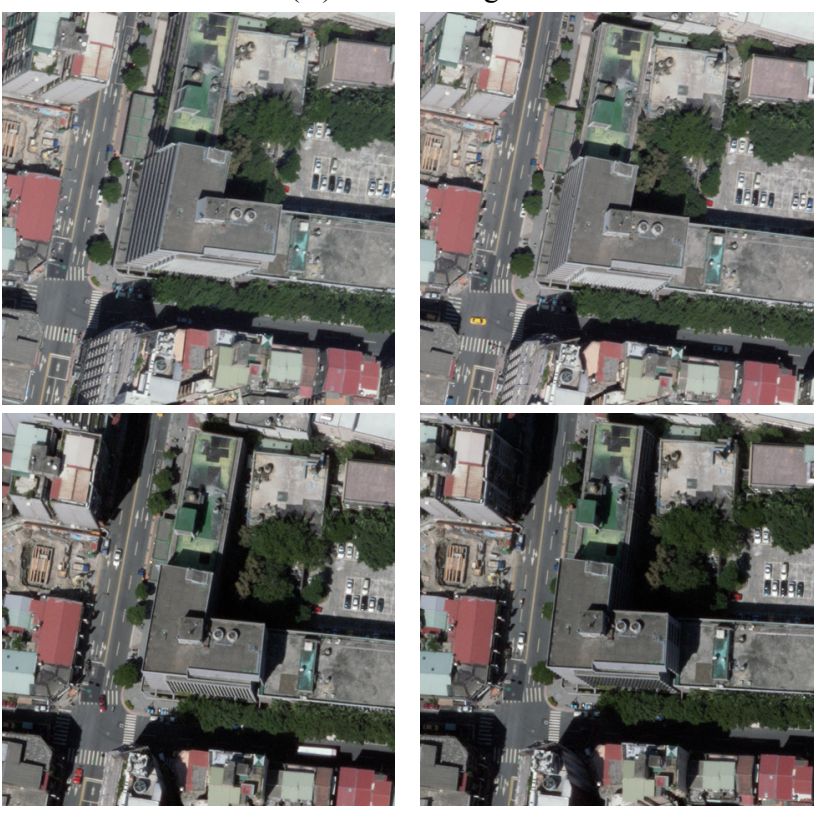

(B) Slave Images

Figure 3. Test Images 
We compared the results of traditional image matching without multi-window, CLR matching, and multi-window matching. Figure 4 shows the results of feature extraction. The total number of feature points is 9947 . The results through those three methods are shown in Table 2. The proposed method provided the highest successful rate. The successful rate of CLR matching is about $64 \%$. And the successful rate is lowest when the image matching was employed without multi-window implementation.

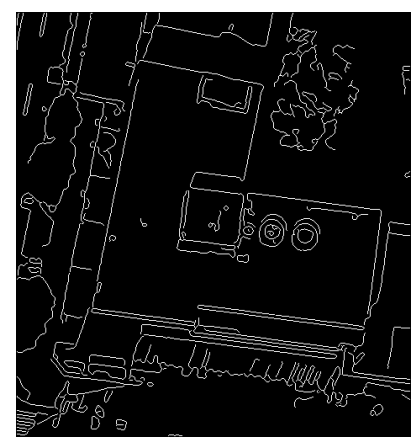

Figure 4. Feature Extraction

\begin{tabular}{|c|c|c|c|}
\hline & $\begin{array}{c}\text { Multi- } \\
\text { window }\end{array}$ & CLR & $\begin{array}{c}\text { without Multi- } \\
\text { window }\end{array}$ \\
\hline $\begin{array}{c}\text { Number of } \\
\text { Successful } \\
\text { points }(\mathrm{R}>0.7)\end{array}$ & 6834 & 6379 & 5328 \\
\hline Successful Rate & $69 \%$ & $64 \%$ & $54 \%$ \\
\hline
\end{tabular}

Table 3. Results of Image Matching

Followed discussions are focused on the building boundaries, which the locations are with elevation discontinuity. We select 30 points to estimate the accuracy of conjugate point pairs in the objective space. The reference data of $3 \mathrm{D}$ coordinates were measured manually.

Figure 5 to Figure 7 show the 3D coordinates of the building from three matching strategies, individually. As one can see, the proposed method can supply the most complete building boundaries. The accuracy of $3 \mathrm{D}$ position results are shown in Table 3. The points with elevation error larger 1 meter are regarded as outliers. Similarly, the proposed method could achieve the highest reliability of image matching. And the root mean square error (RMSE) in elevation direction is small than $0.3 \mathrm{~m}$. In this case, since some edges' directions are near the epipolar direction. It is also observed that the CLR matching might provide the worse results than the image matching without multi-window implementation.
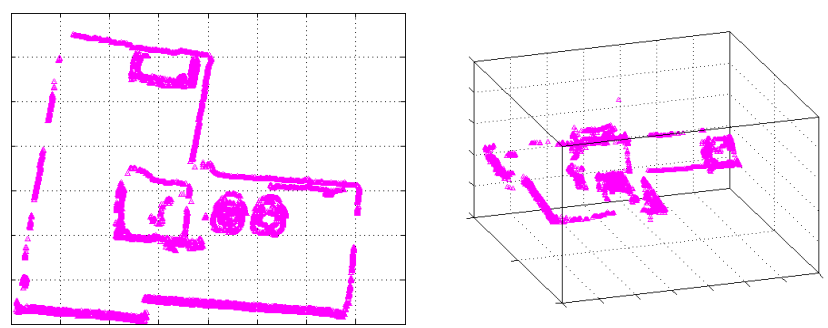

Figure 5. Results of 3D Coordinates by Multi-window
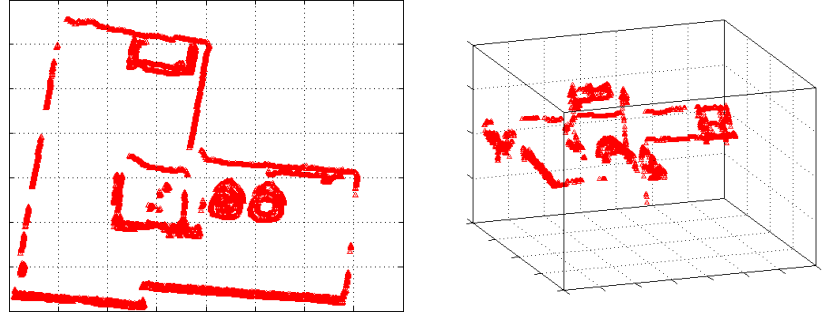

Figure 6. Results of 3D Coordinates by CLR
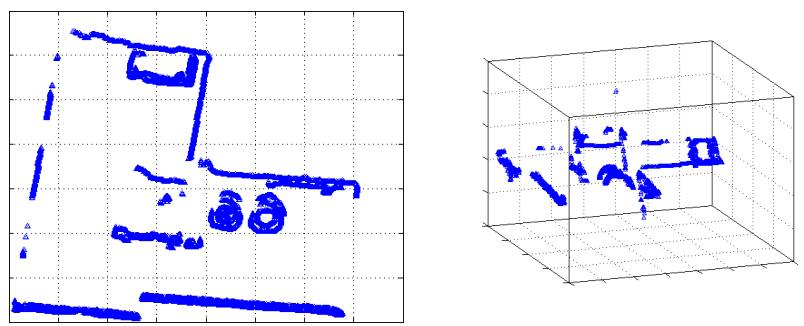

Figure 7. Results of 3D Coordinates by Image Matching without Multi-window

\begin{tabular}{|c|c|c|c|}
\hline & $\begin{array}{c}\text { Multi- } \\
\text { window }\end{array}$ & CLR & $\begin{array}{c}\text { without Multi- } \\
\text { window }\end{array}$ \\
\hline $\begin{array}{c}\text { Number of } \\
\text { Correct Points } \\
(\mathrm{dZ}<1 \mathrm{~m})\end{array}$ & 28 & 21 & 24 \\
\hline RMSE dZ $(\mathrm{m})$ & 0.26 & 0.48 & 0.40 \\
\hline
\end{tabular}

Table 3. Accuracy of 3D Position Results

This paper picks up six points to scrutinize the details of three matching strategies, as shown in Table 4 and Table 5. In Table 4 , the patterns are unlike between master image and slave images. Thus, the correlation coefficients and elevation accuracies are both low in the image matching without multiwindow implementation. The results could be improved by CLR method. But the multi-window matching provides the more dependable and the most correct results.

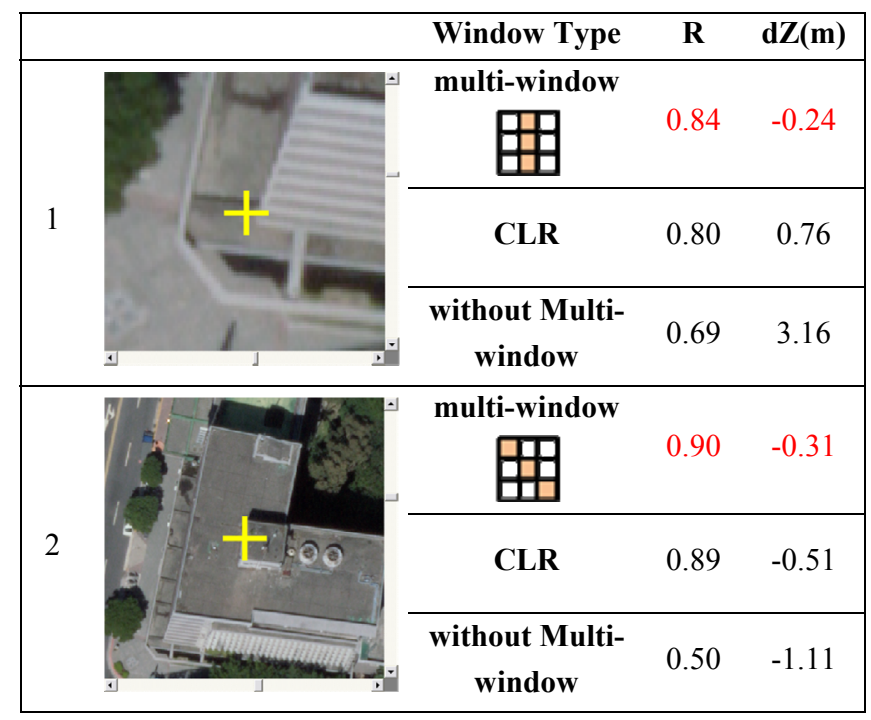

Table 4. Results of Object-based Matching in P1 and P2 
In Table 5, the correlation coefficients are high with no multiwindow strategy in P3 to P6 case. But the conjugate points are outliers because the elevation errors are near even more than 1 $\mathrm{m}$ in $\mathrm{P} 3, \mathrm{P} 5$, and $\mathrm{P} 6$ case. The results become correct through multi-window matching. For P4 and P5 cases, the CLR matching can provide the highest correlation coefficients. But the conjugate points are still outliers. The reason is that the edges' directions are near the epipolar direction, the CLR method, on the contrary, would increase the ambiguous solutions.

\begin{tabular}{|c|c|c|c|}
\hline & Window Type & $\mathbf{R}$ & $d \mathrm{dZ}(\mathrm{m})$ \\
\hline \multirow{3}{*}{3} & $\begin{array}{c}\text { multi-window } \\
\text { PI }\end{array}$ & 0.94 & -0.54 \\
\hline & CLR & 0.94 & -0.54 \\
\hline & $\begin{array}{l}\text { without Multi- } \\
\text { window }\end{array}$ & 0.88 & -0.94 \\
\hline \multirow{3}{*}{4} & multi-window & 0.96 & -0.19 \\
\hline & CLR & 0.96 & -0.19 \\
\hline & $\begin{array}{l}\text { without Multi- } \\
\text { window }\end{array}$ & 0.72 & 0.21 \\
\hline \multirow{3}{*}{5} & & 0.89 & 0.08 \\
\hline & CLR & 0.94 & -1.52 \\
\hline & $\begin{array}{l}\text { without Multi- } \\
\text { window }\end{array}$ & 0.85 & -0.92 \\
\hline \multirow{3}{*}{6} & $\begin{array}{c}\text { multi-window } \\
\text { PH }\end{array}$ & 0.90 & 0.21 \\
\hline & CLR & 0.90 & 1.21 \\
\hline & $\begin{array}{l}\text { without Multi- } \\
\text { window }\end{array}$ & 0.87 & 1.21 \\
\hline
\end{tabular}

Table 5. Results of Object-based Matching in P3 P6

\section{CONCLUSIONS}

This paper analyzed the feature direction first followed by the multi-window matching. We compared the results from different window type. The experimental results indicate that the proposed method improves the accuracy of image matching. It is also observed that the multi-window matching helps locating the building features.

\section{ACKONWLEDGMENTS}

This investigation was partially supported by the National Science Council of Taiwan under Projects No. NSC100-2221E-008-102-MY3.

\section{REFERENCES}

Canny, J., 1986. A Computational Approach to Edge Detection, IEEE Transactions on Pattern Analysis and Machine Intelligence, vol. PAMI-8, pp. 679-698.

Habib, A., Lee, Y. and Morgan, M., 2003. Automatic Matching and Three-Dimensional Reconstruction of Free-Form Linear Features from Stereo Images, Photogrammetric Engineering and Remote Sensing, 69(2):189-197.

Hough, P.V.C., 1959. Machine Analysis of Bubble Chamber Pictures. Proc. Int. Conf. High Energy Accelerators and Instrumentation.

Hsu, W.C., 1999. Building Extraction from Color Aerial Stereo Photo Pairs, Master degree dissertation, National Central University, Taiwan. (In Chinese)

Rosenholm, D., 1987. Least-squares Matching Method: Some Experimental Results, Photogrammetric Record, 12(70):439512.

Xiong, Z., and Y. Zhang, 2009. A Novel Interest-PointMatching Algorithm for High-Resolution Satellite Images, IEEE Transactions on Geoscience and Remote Sensing, 47(12):4189-4200.

Zhang, L., and A. Gruen, 2006. Multi-image matching for DSM generation from IKONOS imagery. ISPRS Journal of Photogrammetry and Remote Sensing, 60(3):pp. 195-211. 